\title{
Development of an Underwater Robot for Sediment Soil Sampling
}

\author{
Peng Gao ${ }^{1,}$, , Bin Zheng ${ }^{1, b^{*}}$, Jianhong Liang ${ }^{2, c}$ and Cangsi Ren ${ }^{2, d}$
}

${ }^{1}$ Chongqing Key Laboratory of Multi-Scale Manufacturing Technology, Chongqing institute of Green and Intelligent Technology, Chinese Academy of Sciences, Chongqing, China

${ }^{2}$ Beihang University, Beijing China

agpeng@cigit.ac.cn, bzhengbin@cigit.ac.cn, ${ }^{\mathrm{c}}$ dommy_leung@263.net, ${ }^{\mathrm{d}}$ rcs@aheadx.com

Keywords: sediment soil; sampler; robot; ROV.

Abstract. An underwater sediment soil sampling robot is presented in this paper. The developed robot is composed of a Remotely Operated Vehicle (ROV), a buoyancy regulator, a sampling corer and other parts. Differ from traditional ROVs and sampling devices, the robot moves by swinging the fins and collects soil by regulating the buoyancy. This robot keeps neutral buoyant before sampling. When it is sampling, the buoyancy regulator is activated to drive the robot to dive and insert the sampling tube into the soil. The sampling corer is optimized to suit the robot. Experiments for evaluating the motion performance of the robot is explained and the field sampling is accomplished. The sampling result proves the utility of the robot in sediment soil sampling field.

\section{Introduction}

Sediment soil sampling plays an important role in water environment monitoring. Scientists can obtain valuable components information and observe the microbe species by analyzing the sampled soil to understand various phenomena in geochemistry, biology and marine science [1,2]. In the past decades, sampling technology research gets growing attention [3, 4] and various types of samplers for collecting sediment soil bloom [5]. Among of all kinds, core samplers are widely used. The sampler is put into water and sinks until the frame landing on the bottom, then the sampling core continues sinking and inserts into the sediment soil. However, core samplers are gravity samplers leading to an inconveniently operating weight and have a low success rate cause of non-maneuverability.

Robotic technology is used for soil sampling in recent years [6], by controlling a Remotely Operated Vehicle (ROV), scientists are easy to obtain the sediment soil for research. In this paper, an underwater sediment soil sampling robot is proposed.

The outline of this paper is as follows: Firstly, all components of the sampling system are introduced. Next, configuration of the robot is presented. Then the details of the buoyancy regulator and the core sampler are carried out. Furthermore, a motion evaluation test is recorded for evaluating the movement performance of the robot. Finally, a sampling method is illustrated and an experiment of the developed robot accomplishing the sampling task in a river is shown.

\section{Illustration of Robot System}

As shown in Fig. 1, the sampling system consists of an offshore platform (or a ship), a remote computer, an underwater robot, a couple of buoyancy regulator, a set of sampler, a crane, a cord reel, an umbilical cable and an anchor. The umbilical cable connecting the remote computer and the robot is neutral buoyant. Control instructions sent from the computer by an operator using a joystick and sensor information from the robot transform through the cable. The crane is used to lift and lower the robot. The cord reel will roll the cable when the crane is being operated. The buoyancy regulator and the sampler are mounted on the robot. The buoyancy regulator changes the buoyancy of the robot to obtain the drive force for inserting the sampling corer into the sediment soil. 


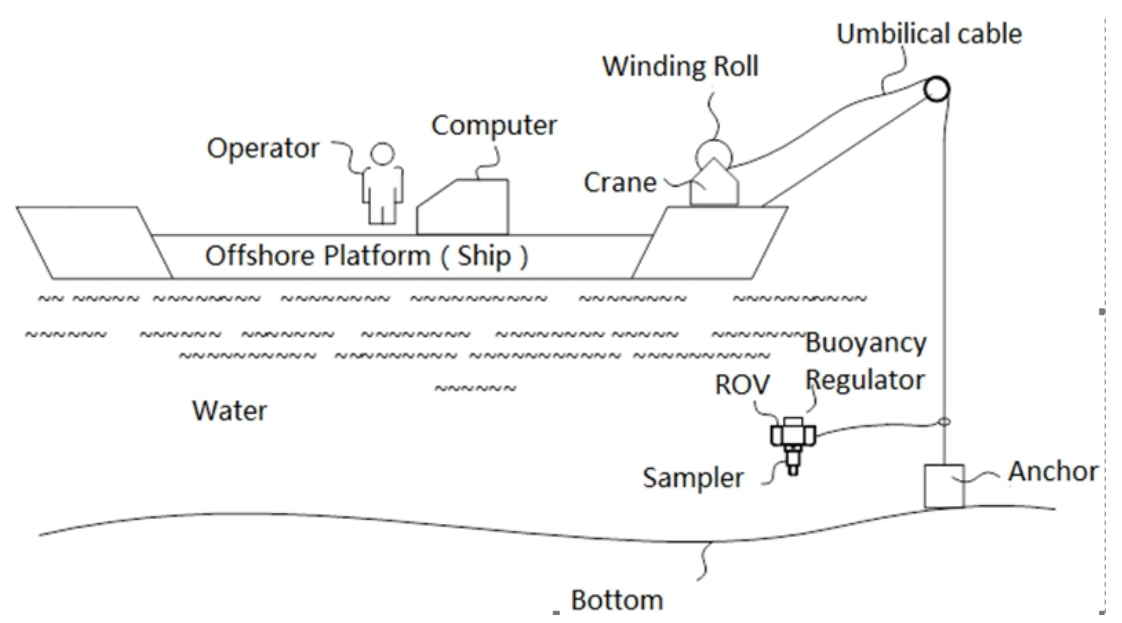

Fig. 1 Components of the sediment soil sampling system

\section{Design of the Robot}

A four-fin-thruster underwater robot is developed for soil core sampling as shown in Fig. 2. In this section, the construction of the robot is described.

Main Body. The main body is a hollow cylinder tubular with both ends smoothed and coned to reduce resistance. To contain electrical devices including power batteries, controllers for data communication and thrust controlling, a camera, DC servomotors and motor drivers, sensors and other components, the hollow housing is designed as $1.0 \mathrm{~m}$ long, $0.2 \mathrm{~m}$ in diameter. Meanwhile, waterproof and pressure-tight design considered.

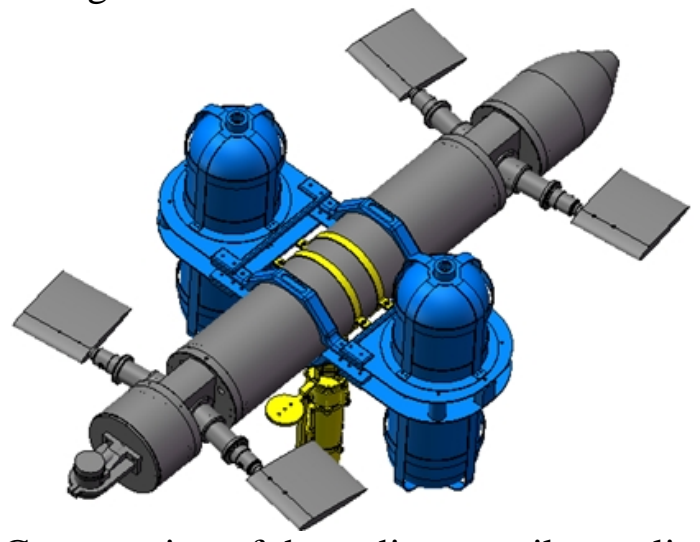

Fig. 2 Construction of the sediment soil sampling robot

Thruster. Differ from traditional underwater thrusters, the four-fin configuration is used for thrusting the robot. Each thruster is a fin-like board driven by a 90W DC servomotor with a gearbox of which the transmission ratio is 40 . When the four boards swinging at different angles, frequencies or ranges, the robot moves in different ways such as forward and back, up and down, roll, pitch yaw and hover.

Sonar and Camera. A sonar is installed on the front end of the robot for obstacle avoidance. The sonar is controlled by the remote computer to scan the water environment and sends data to the computer. The operator observes the image shaped from the data to judge if the robot is facing obstacle and controls the robot move depending on the analyzing results.

There is no enough light in the deep water, it has a very bad visibility for the camera to catch photos. A camera with a major light is mounted under the robot to monitor the sampler and records the sampling process.

Control Strategy. A Microcontroller Unit (MCU) is inside the developed robot and communicates with the remote computer via a 50m long umbilical cable. Motion instructions are sent from to the robot the computer by an operator using a joystick. When the robot receives instructions, the MCU automatically analyzes them and controls the robot to move according to the programmed codes and parameters. 


\section{Buoyancy Regulator and Sampler}

Craib [7] proposed a well-known core sampler named Craib corer in 1965. Driven by lead weights, the Craib corer slowly lowers a tube into the sediment under the control of a hydraulic damper. In this paper, a buoyancy regulator is taken place of the lead and the hydraulic damper. When sampling instruction is given, the sampler is inserted into the sediment soil by the vertical downward force from the result of the buoyancy regulating.

Buoyancy Regulator. The buoyancy regulator is made up of two diving gas bottles installed on both sides of the robot. The two bottles can bear the pressure up to $30 \mathrm{MPa}$ and have the volume of 13.6L. A solenoid valve is connected on the nozzle upon each bottle. Compression strength of the used valves are designed up to $30 \mathrm{MPa}$. Until the sampling starts, bottles are filled with air gas and solenoid valves are OFF. When the valves are turned $\mathrm{ON}$ by the remote computer, the water will go into the bottles and the gas bubbles will rise, thus, the buoyancy of the robot will be negative and begin to sink.

Soil Sampler. A new soil sampler based on the principle of the Craib corer is shown in Fig. 3. The sampling tube is made of acrylic and is transparent for observation, other parts are stainless steel. Two rings fix the tube and are connected by a linkage which is also the base of the bottom catcher mechanism. The bottom catcher arm is made up of a spring-loaded sliding rod, a short double-jointed linkage and a rotary rod articulated. The upper ring is connected by another linkage with the outside of a cylindrical ring whose inner surface is amounted on a cylinder. The top catcher is installed upon the upper ring with four evenly distributed smooth round bars sliding through the pores outside of the ring. Three spring clips are radiating installed on the cylindrical ring and are tensed by a protrusion-amounted cylindrical ring. Two pins in the two of the three protrusions are used to preventing the cylindrical rotating and limiting the sliding range. The three clips are hoop-designed and two special parts are amounted on the both catchers to suit the hoop clips, one clip holds the bottom catcher and the other two hold the top catcher.

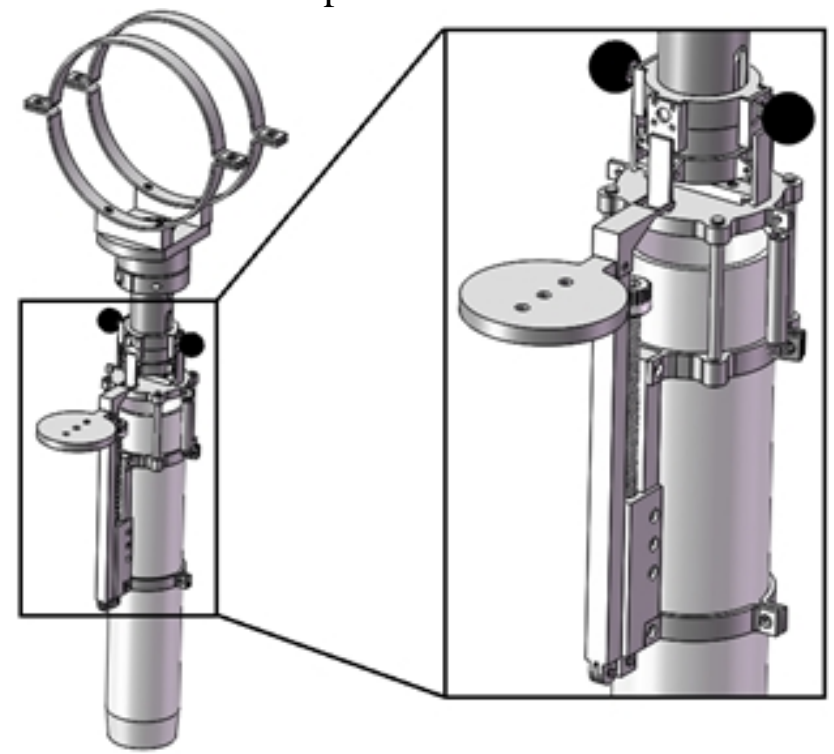

Fig. 3 Construction of the sampler

Springs loaded on the top catcher, the bottom catcher and the clips are all tensed when the protrusion-amounted cylindrical ring is posited as Fig. 3. In the sampling process, after the tube inserting into the soil, the cable will be dragged by the remote crane, the protrusion-amounted cylindrical ring is pulled sliding upward until the pins is limited. At the instant the protrusion no longer supports the clips, the two catchers are released by the clips. The top catcher slides downward and closes the top end of the tube, meanwhile, the bottom catcher swings down through about $90^{\circ}$ and resets on the bottom. Then the tension of the cable is growing, as a result, the whole robot is pulled up, the sampler is pulled out of the bottom slowly and the bottom catcher is continuing to swing. When the tuber is off the river bed completely, the bottom end will be totally closed by the bottom catcher. 
Owing to the negative pressure from the top catcher sealed closing and the viscosity of sediment soil, the sediment soil will not drop during the process of pulling the tube out of the bottom.

\section{Motion Performance Evaluation}

The motion performance of the developed robot is resented via a serial of tests in a swimming pool. In the tests, the robot receives motion instructions sent from the remote computer, and the MCU in the robot processes the data and controls the robot to move actually as the required motion. In this paper, the performance test of hover capacity is carried out.

First, the robot is released floating under the surface of the water and the depth senor is located at the depth of $0.1 \mathrm{~m}$. Then the goal depth of $0.5 \mathrm{~m}$ is sent to the robot by the operator. The robot receives the instruction and dives until the actual depth is equal to the goal depth. The process of the moving and hovering is recorded as Fig. 4, the adjustment of the depth spends about 8s.

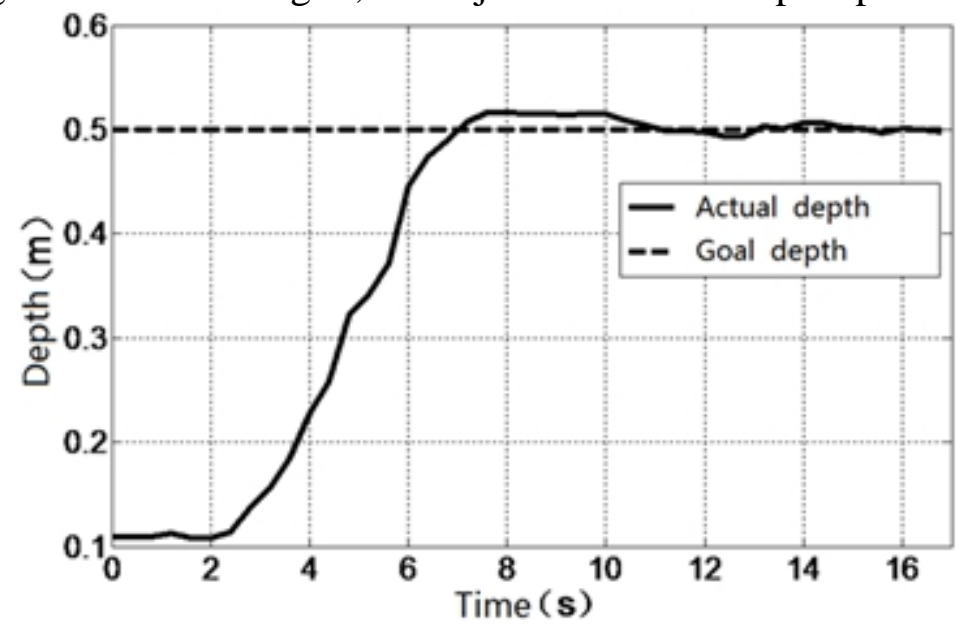

Fig. 4 Performance of the robot hovering

\section{Field Sampling and Result}

Backwater zone of the Pegnxi River belonging to the Three Gorges Reservoir Area where the robot is planned to use was chosen as the sampling field. In the experiment, the robot dived three times at different area under the control of the remote computer operator with a joystick, and collected three tubes of sediment soil from the bottom of the river of which the depth is about $18 \mathrm{~m}$ depth.

Fig. 5 shows the developed underwater sediment soil sampling robot tested in the sampling field and the photo when it was diving for sampling.
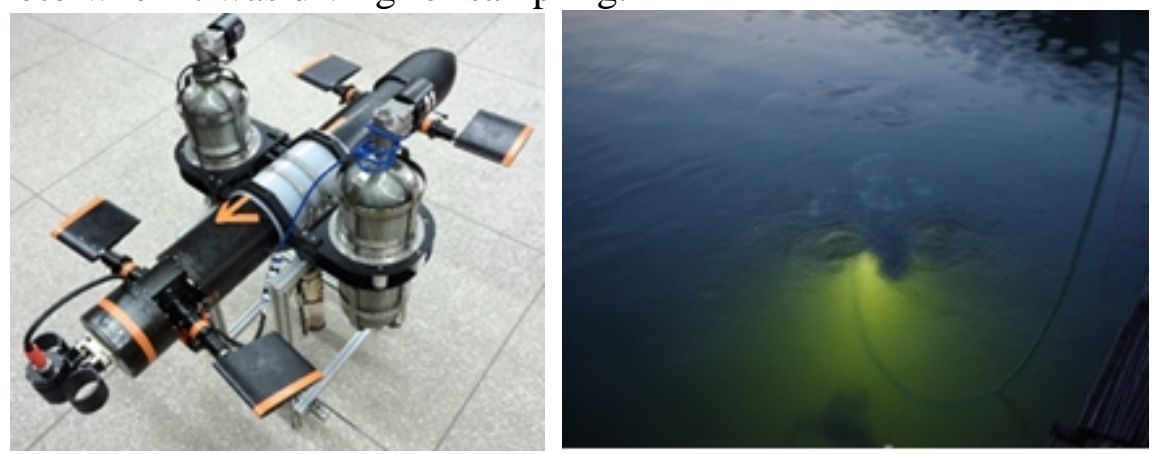

Fig. 5 The developed robot

The schedule of the robot sampling is as follows.

1) Sinking the robot: The operator controls the crane to sink the robot and the anchor into the water, the anchor dives by its weight, meanwhile the robot is driven to dive by the fin boards controlled by the MCU. 
2) Landing on the bottom: The anchor keeps diving until reaching the bottom of the water. The robot begins to swim in a circle with the landing point of the anchor. The anchor bears the force son the cable from the water current, so that the robot just needs to overcome the motion resistance.

3) Locating the sampling field: The robot keeps swimming in the scope of the circle and the video by the camera is been sending to the remote computer, the operator observes the video and selects the adaptive area for the robot sampling.

4) Sampling soil: After the sampling area is located, the robot hovers with a suitable posture, the valves of the buoyancy regulator are turned $\mathrm{ON}$, the gas bubbles rises and water inflows. Now, the buoyancy of the robot is no longer neutral and a vertical negative force grows driving the robot down to insert the tube into the sediment soil.

5) Catching soil: By the camera amounted on the robot, the operator estimates the sampling process. Once the sampling is accomplished, the cable is dragged by the crane, the sampler is triggered, and the catchers held by springs are released. The top catcher is closed on the top end of the sampling tube, at the same time the bottom catcher is also swings and is prevented by the bottom of water. The cable continues to be dragged, the robot rises and the tube is slowly lifted out of the bottom. At the moment the tube is totally off the river bed, the bottom catcher closes. Thus the sediment soil is successfully caught.

6) Taking back the robot: The cable keeps been dragging until the robot is lifted out of the water, operators on the offshore board take back the robot and detach the sampler, the sampler is sent to environmentalists for researching the sediment soil inside. Hereto, the sediment soil sampling is accomplished.

Fig. 6 displays the process of the sediment soil sampling recorded by a camera amounted under the robot. Fig.7 shows the collected sediment soil. The collected three tubes of soil have been sent to environmentalists for the composition analysis.

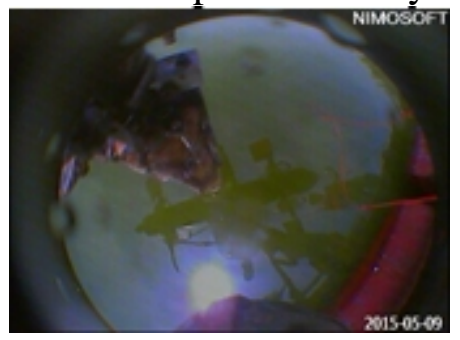

a. Before dive into the water

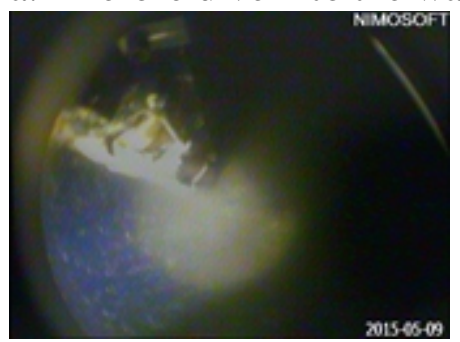

c. Reaching the bottom

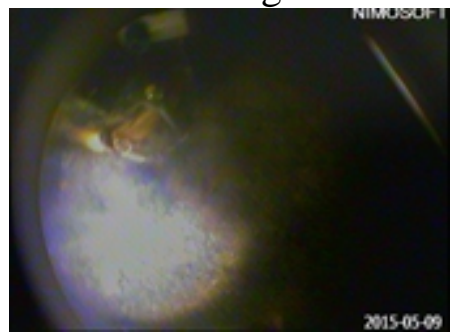

e. Finishing the sampling

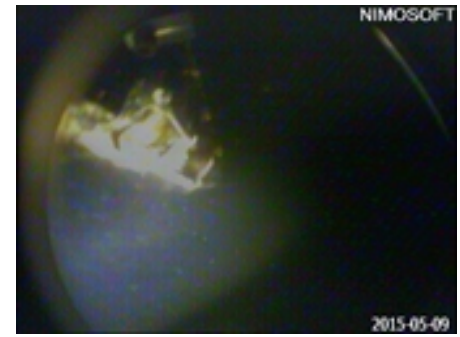

b. Diving to the bottom

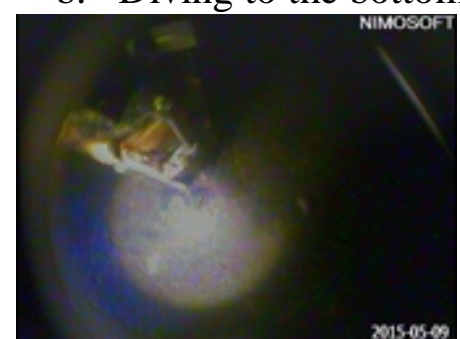

d. Inserting the soil

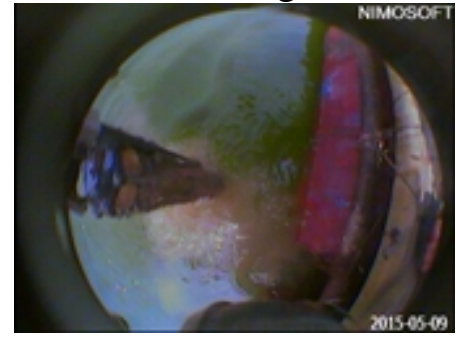

f. Out from the water

Fig. 6 Process of the sediment soil sampling 

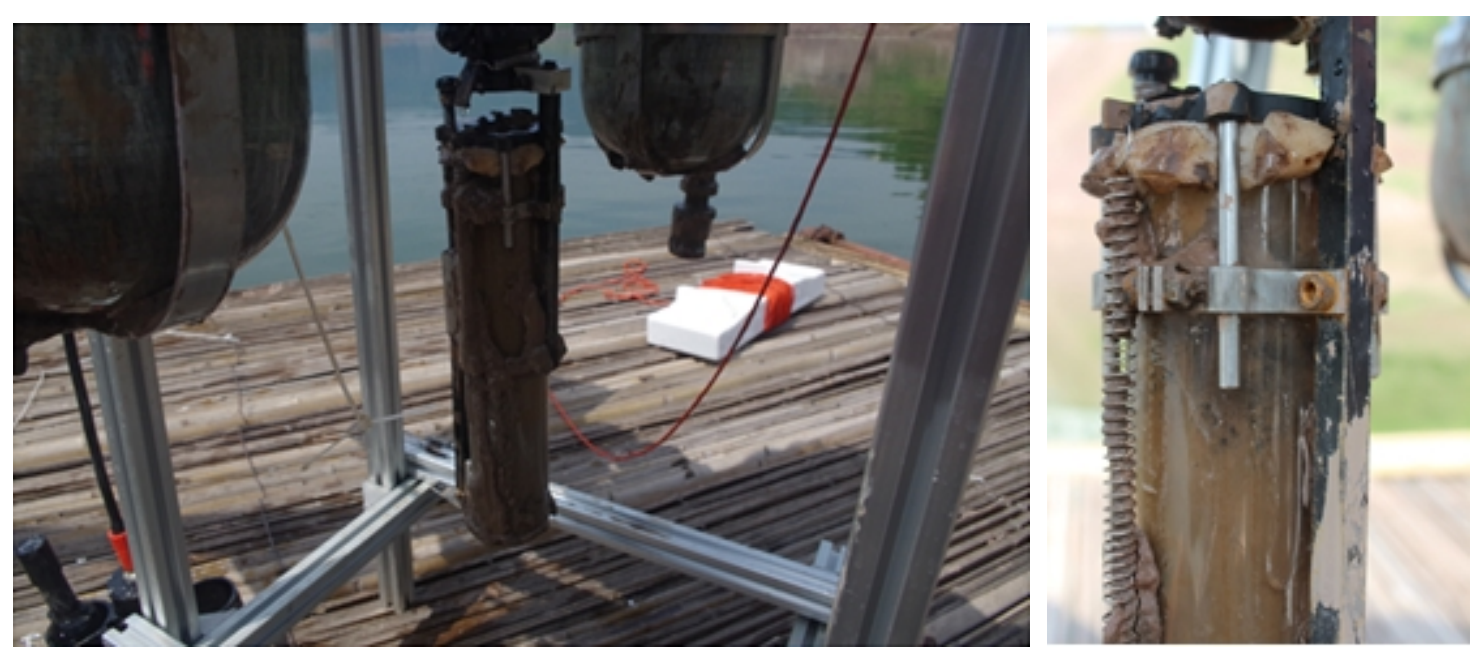

Fig. 7 The collcoted sediment soil

\section{Summary}

A sediment soil sampling robot is described and a sampling method is introduced in this paper. The developed robot takes fin-like boards instead of propellers as thrusters. It keeps neutral-buoyancy to move in water and changes the net buoyancy using the regulator to obtain a driving force to collect the sediment soil. The motion performance is shown and the sampling experiment is taken to prove the utility of the robot in sediment soil sampling field.

As future works, the robot will be taken to the Three Gorges Reservoir Area to sample the sediment soil where the depth is above 100 meters.

\section{Acknowledgement}

This paper is supported by Chongqing Robotic Industry Development of Overall Plan, Key Technology Import and Innovation Ability (No. cstc2013jcsf-zdzxqqX005), and Bin Zheng is the corresponding author.

\section{References}

[1] Xueqiu Wang, the CGB Sampling Team, China geochemical baselines: Sampling methodology, J. Journal of Geochemical Exploration. 148(2015) 25-39.

[2] Pierrick Bocher, Theunis Piersma, Anne Dekinga, Casper Kraan, Michael G. Yates, Thierry Guyot, Eelke O. Folmer, Gilles Radenac, Site- and species-specific distribution patterns of molluscs at five intertidal soft-sediment areas in northwest Europe during a single winter, J. Marine Biology. 151(2006) 577-594.

[3] L.C. Skinner, I.N. McCave, Analysis and modelling of gravity- and piston coring based on soil mechanics. Mar Geol 199 (2003) 181-204.

[4] Wojciech Dabrowski, R. Ian Mackie, Michal Zielina, Does Freezing Affect Sediment Sampling Results? J. Water, Air, and Soil Pollution. 140(2002) 367-370.

[5] Barnett P.R.O., Watson J., Connelly D., A multiple corer for taking virtually undisturbed samples from shelf, bathyal and abyssal sediments, J. Oceanol Acta. 7 (1984) 399-408.

[6] Sakagami N., Sasaki S., Kawabata M., Yokoi K., Matsuda S., Mitsui A., Sano K., Tago K., Kawamura S., Development of a human-portable underwater robot for soil core sampling, C. OCEANS - Bergen, 2013 MTS/IEEE 1 - 6.

[7] Craib J. S., A sampler for taking short undisturbed marine cores, J. Cons. Perm. Int. Explor. Mer. 30(1965) 34-39. 